\title{
... $e^{+} e^{-}$REACTIONS - EXPERIMENTS*
}

Rudolf R. Larsen

Stanford Linear Accelerator Center

Stanford University, Stanford, California 94305

\section{ABSTRACT}

We present current results of the SLAC-LBL survey experiment** on the annihilation of $\mathrm{e}^{+} \mathrm{e}^{-}$into hadrons in the $s$ range $6 \mathrm{GeV}^{2}$ to $25 \mathrm{GeV}^{2}$.

\section{INTRODUCTION}

The experimental study of $\mathrm{e}^{+} \mathrm{e}^{-}$annihilation into hadrons has proceeded from the elegant studies of vector meson production performed at ACO (Orsay) and NPI (Novosibirsk) to the pioneering work on inelastic hadron production at ADONE (Frascati) and CFA (Cambridge) and now to the current experiments at SPEAR. The field is expanding rapidly as it has become apparent that it is a critical testing ground for the most basic concepts of elementary particle interactions.

This discussion will consider, with few exceptions, the current results of the SLAC-LBL experiment at SPEAR. The topics discussed are: Section $I$, the experimental apparatus; Section II, a brief summary of QED tests; Section III, measurements of $\sigma_{\mathrm{T}}$, the total cross section for annihilation into hadrons; Section $T V$, general features of the hadronic final states; Section $V$, inclusive hadronic spectra and tests of scaling; Section VI, current conclusions and comments on future directions. \footnotetext{
* Work supported by U. S. Atomic Energy Commission

Participants: (Stanford Linear Accelerator Center)

J.-E. Augustin, A. M. Boyarski, M. Breidenbach, F. Bulos,

J. T. Dakin, G. J. F'eldman, G. E. Fischer, D. Fryberger,

G. Hanson, B. Jean-Marie, Rudolf R. Larsen, V. Iuth,

H. I. Lynch, D. Lyon, C. C. Morehouse, J. M. Paterson, M. Perl,

T. Pun, B. Richter, R. F. Schwitters, F. Vannucci.

(Lawrence Berkeley Laboratory) G. S. Abrams, D. Briggs,

W. Chinowsky, C. E. Friedberg, G. Goldhaber, R. J. Hollebeek,

J. A. Kadyk, F. Pierre, G. H. Trilling, A. Wagner, J. S. Whitaker,

J. E. Zipse.
} 


\section{THE EXPERTMENT}

The SPEAR magnetic detector commenced taking data in Sept. 1973. The apparatus, depicted in Fig. 1, consists of a $3 \mathrm{~m}$ diameter, 3-m long solenoid, axis coincident with the storage ring beam line and generating a uniform field of $4 \mathrm{kG}$. A particle leaving the interaction region will radially traverse in sequence the vacuum chamber, a pipe scintillator, 16 cylinders of magnetostrictive wire spark chambers which provide the track information, a cylindrical array of 48 scintillators (the trigger counters), the one radiation length magnet coil, a cylindrical array of 24 lead-scintillator shower counters which provide electron identification, the $20 \mathrm{~cm}$ iron flux return and finally two gaps of plane spark chambers.

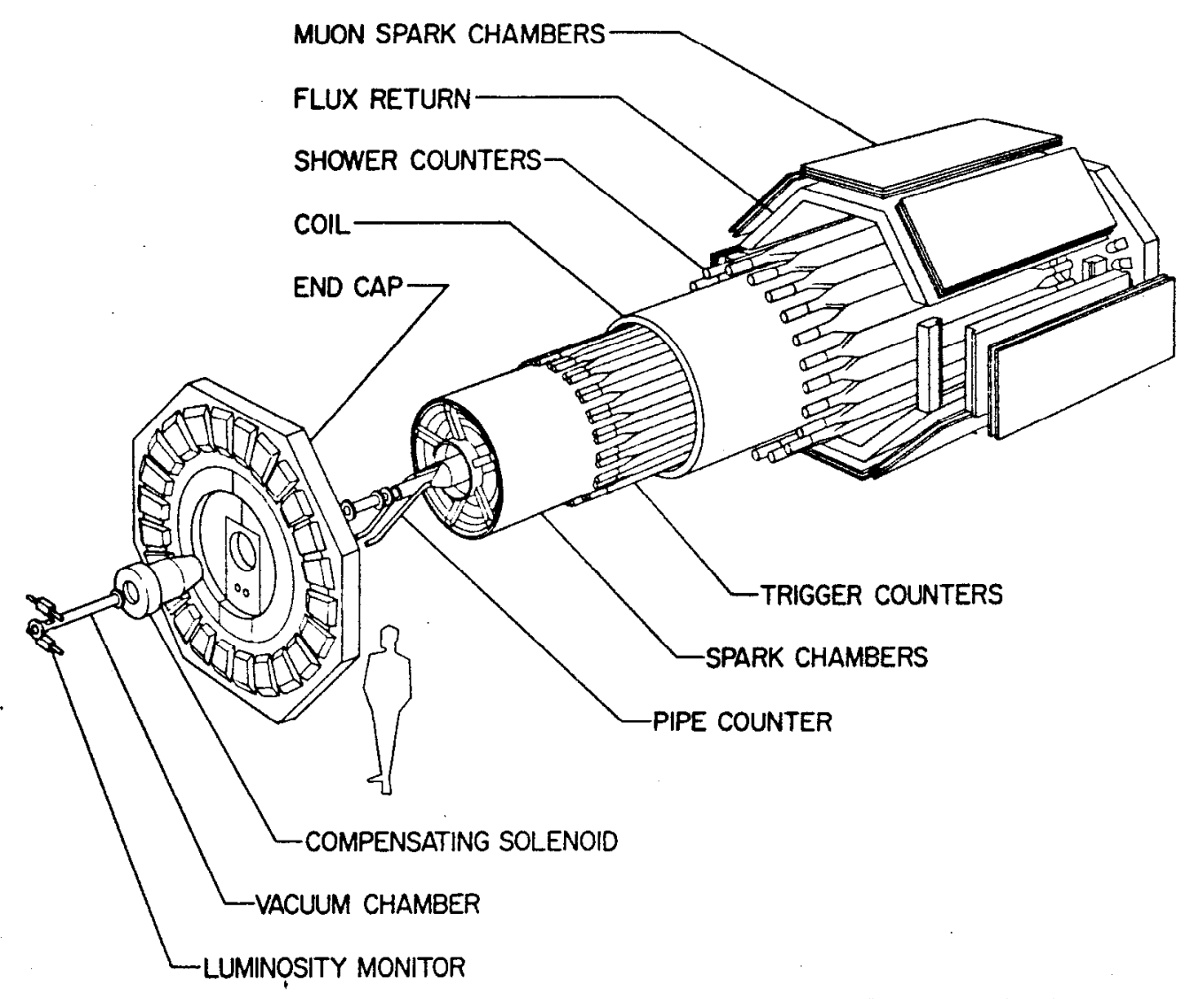

FIG. 1--Magnetic detector telescoped along axis for viewing.

The trigger counters and shower counters subtend a solid angle of $0.65 \times 4 \pi$ ster. about the interaction region. The measurement resolution for a single unconstrained track is $\Delta \varphi$ (aximuth) $= \pm 5 \mathrm{mrad}$, $\Delta \theta($ polar $= \pm 5 \mathrm{mrad}, \Delta \mathrm{p} / \mathrm{p}= \pm 2-9 \%$ as the momentum varies from 0.5 to $2 \mathrm{GeV} / \mathrm{c}$.

Phototube times, pulse heights and spark-chamber wand pulses are digitized and logged on tape whenever the basic trigger requirement is satisfied. A sample of these events are immediately analyzed 
and provide an on-line diagnosis of the detector status. The trigger is a time coincidence between the passage of the beam bunch, a pulse from the pipe scintillator and pulses from at least two independent trigger counter-shower counter combinations. The acquired events are analyzed into the broad classifications: cosmic rays ( 40\%), QED and hadronic events ( 20\%), background $(\sim 40 \%)$. Background subtractions are made by collecting data with single or separated beams and normalizing to the integrated pressure times current; backgrounds subtracted from the hadron and QED samples amount to about $5 \%$.

Hundreds of hadronic events have been collected at beam energy intervals of $0.1 \mathrm{GeV}$ ranging from $1.2 \mathrm{GeV}$ to $2.5 \mathrm{GeV}$. Thousands of events were acquired at emphasized energies of $1.5,1.9$, and $2.4 \mathrm{GeV}$. The general results of the experiment are not statistically limited.

\section{QED TESTS}

In this experiment QED is tested by comparing theory and observations of the angular distribution of Bhabha scaitering, $\mathrm{e}^{+}+\mathrm{e}^{-}$ $\rightarrow e^{+}+e^{-}$and $e^{+}+e^{-} \rightarrow \mu^{+}+\mu^{-}$. The reader is reminded that Bhabha scattering involves a (momentum transfer) ${ }^{2}$ of $\mathrm{q}^{2}=+s$ (time-like) and $q^{2}=-s(\sin \theta / 2)^{2}$ (space-like) while muon production involves only $q^{2}=+s$. In the conventional manner, the theory is modified by including a form factor $F\left(q^{2}\right)=\left(I \pm q^{2} / \Lambda^{2}\right)$ with each amplitude and comparing the radiatively corrected predictions with observation.

QED events are selected by imposing the cuts: (1) net charge of zero, (2) two tracks collinear to $\leq 10^{\circ},(3)\left|\cos \theta_{+}\right| \leq 0.5$ to eliminate edge effects, (4) momentum of each track $2 \mathrm{p}_{\max } / 2$ and (5) a cut on the sum of the two shower counter pulse heights as displayed in Fig. $2^{*}$.

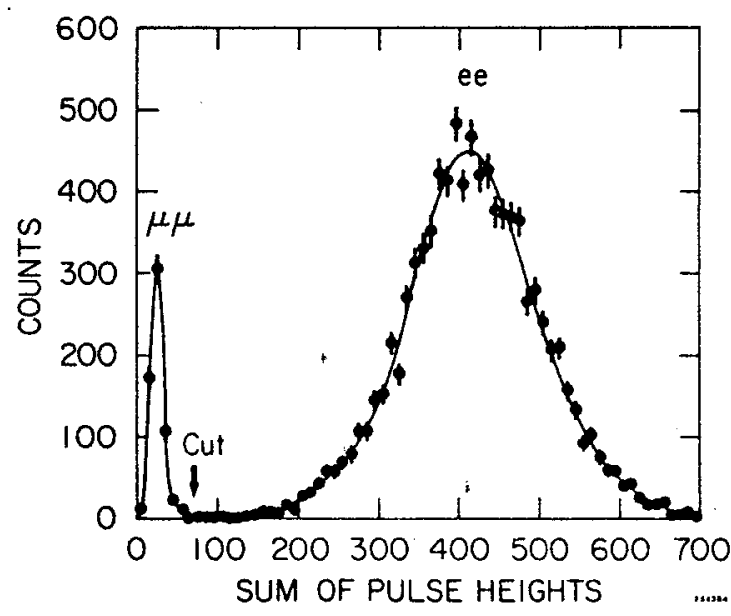

FIG.2--Shower counter pulse heights for collinear events at $s=23 \mathrm{GeV}^{2}$.

*AIl figures presented in this paper must be considered to be of a preliminary nature. 


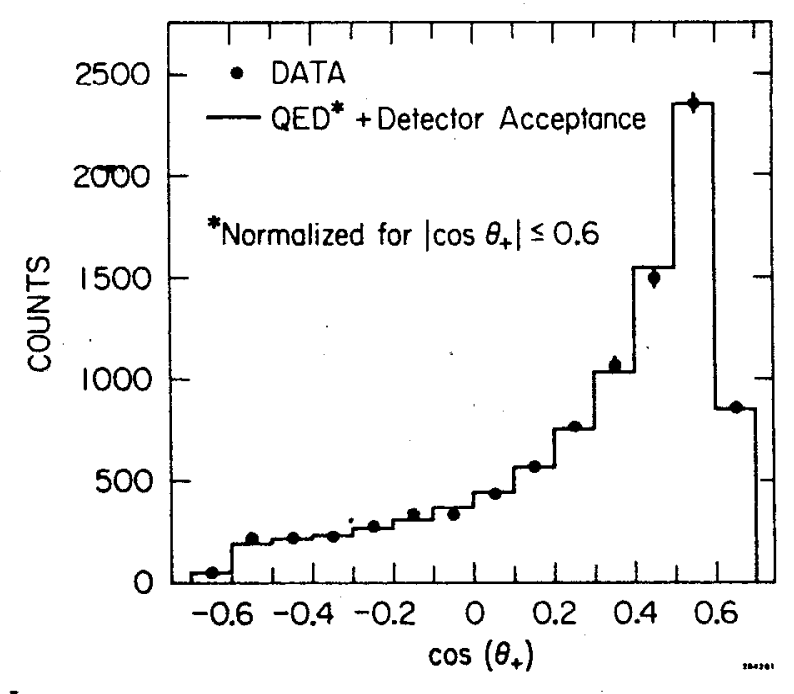

FIG.3--Angular distribution of Bhabha events at $s=23 \mathrm{GeV}^{2}$.
Figure 3 shows, for example, the observed and predicted $(\Lambda \rightarrow \infty)$ angular distribution for Bhabhas at $\mathrm{s}=23 \mathrm{GeV}^{2}$. The comparison is made at $s=9,14.4$ and $23 \mathrm{GeV}^{2}$ and the results combined to give the minimum values (95\% C.I.) of the $\Lambda^{\prime}$ s as sumarized in Table $I$. While more extensive analysis has been done, the particular values quoted here corre: spond to modification of the photon propagator and assume no vertex modification and equal space-like and timelike $\Lambda$ 's. QED successfully meets another test!

Table I

Minumum Values ( $95 \%$ C.L.) of $\Lambda$

\begin{tabular}{|l|c|c|}
\hline Parameter & \multicolumn{2}{|c|}{ Data } \\
\hline \multirow{4}{*}{$\Lambda_{+}(\mathrm{GeV})$} & $\mathrm{e}^{+} \mathrm{e}^{-}$ & $\mathrm{e}^{+} \mathrm{e}^{-}$and $\mu^{+} \mu^{-}$ \\
\cline { 2 - 3 }$\Lambda_{-}^{+}$ & 16 & 35 \\
& 13 & 33 \\
\hline
\end{tabular}

Henceforth, all cross sections reported in this paper are deduced by normalizing yields to the number of Bhabha events concurrently observed.

\section{TORAL ANNIHILATION CROSS-SECTION vs. s}

We will discuss this aspect of the experiment in three steps: (1) the raw data as a function of $s$ (2) the detection efficiency and (3) the total cross section and corrections thereto.

\section{The Data}

Hadronic events are selected by requiring that the event vertex be located within a cylindrical volume of $4-\mathrm{cm}$ radius and $80-\mathrm{cm}$ length. (Two prong events with net charge zero were further required to be acoplanar by at least $20^{\circ}$ and to be minimum ionizing; see Sec. III, 3.) Contributions not originating from beam-beam interactions were subtracted as described in Sec. I. Figure 4 is a plot of the vertex $z$ distribution for colliding and noncolliding configur- 


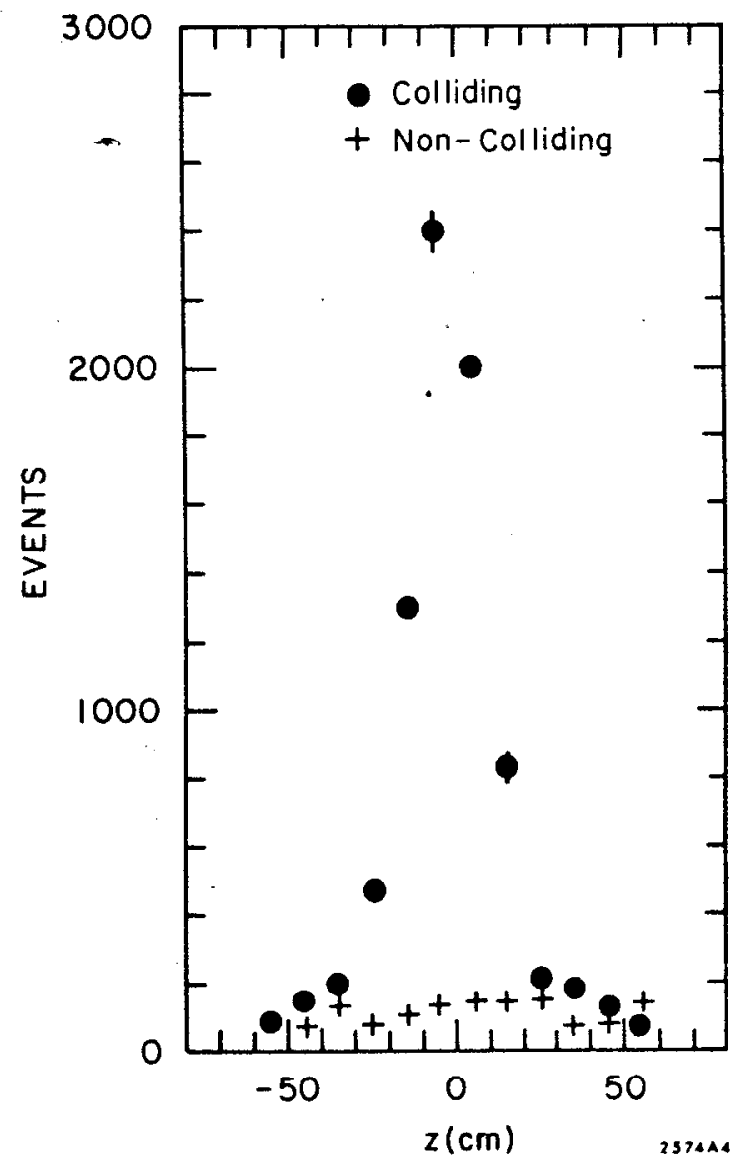

FIG. 4--Vertex $\mathrm{z}$ distribution at $\mathbf{s}=23 \mathrm{GeV}^{2}$.

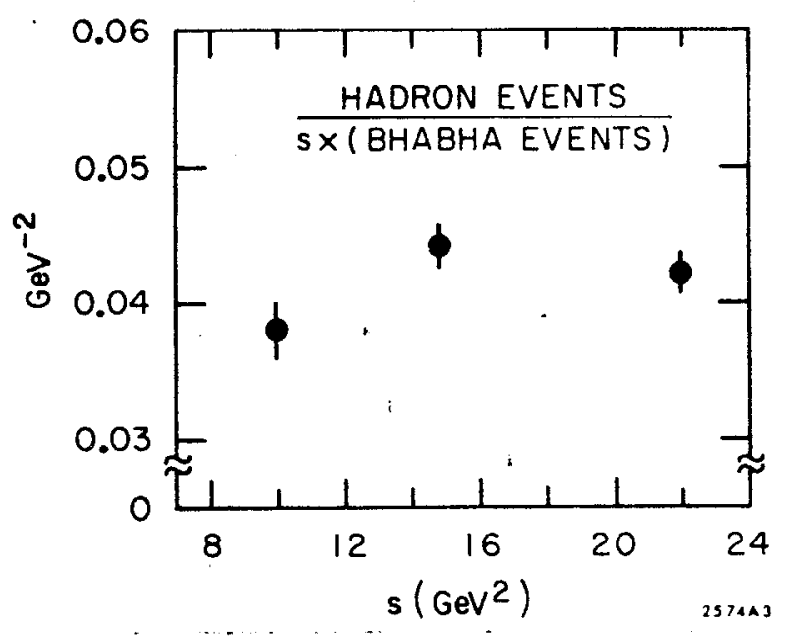

ations at $s=23 \mathrm{GeV}^{2}$. The events thus selected at $s=9$, 14.4 and $23 \mathrm{GeV}^{2}$ are divided by $s$ times the number of concurrently observed Bhabha events to yield a quantity proportional to the uncorrected hadronic cross section; results are plotted in Fig. 5 with statistical errors. Clearly, there is little $s$ dependence present.

\section{The Detection Efficiency}

The quantity displayed in Fig. 5 must now be corrected to account for the inefficiency of the apparatus to detect hadronic events. It is apparent that if the charged multiplicity, mean momenta, and angular distribution do not change rapidly with $s$, then neither will the detector efficiency. Furthermore, because of the large detector acceptance, rapid changes in the above produced parameters will be reflected in the

FIG. 5--Ratio of observed hadronic events to $s$ times the observed Bhabha events. 
corresponding observables. Figure 6 displays $\left\langle\mathrm{n}_{\mathrm{ch}}\right\rangle$ and $\langle\mathrm{p}\rangle$ observed vs. s. It will be seen later that the angular distributions are essentially isotropic at all $\mathrm{s}$. With this information one won't be surprised if the efficiency is not very sensitive to $\mathrm{s}$.

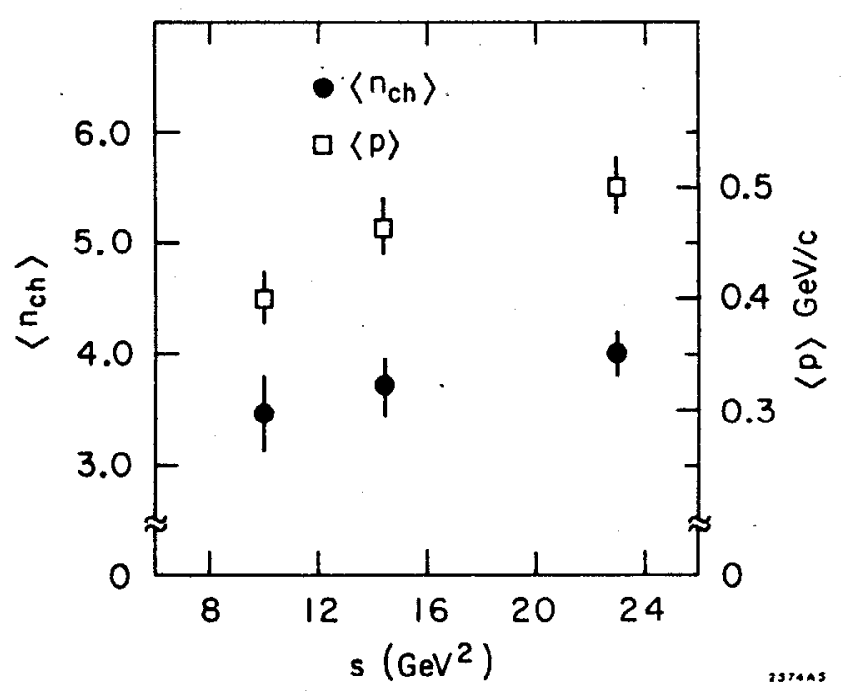

FIG. 6--Mean multiplicity and track momentum observed.
Calculation of the efficiency necessarily involves a model of the final states: we have assumed that only charged and neutral pions are present and are distributed in angle and momentum according to a relativistically invariant phase space. Total multiplicity is assumed Poisson and charged and neutral distributed binomially within the total. The events are generated in a computer simulation of the magnetic detector which includes a.l known properties of beams, detector and observed event selection that could result in ineffi-

ciencies. The mean total multiplicity and the factor determining the charged to neutral ratio are constrained to match the observed mean momentum and multiplicity, respectively. Once these parameters are fixed, the average efficiency is calculated as the ratio of events that should be observed to those that are generated. We go further and remove the assumption of a Poisson distribution by using calculated partial probabilities to observe $m$ tracks when $n$ are produced; these are used in a set of equations relating observed and true multiplicities to deduce the latter and then calculate an event efficiency as the average of the partial efficiencies weighted by the true multiplicity. This method, called the UNFOLD, produces results that differ by at most $10 \%$ (and usually less than 5\%) from the Poisson assumption. The average efficiency deduced from the UNFOLD is displayed in Fig. 7 and is, as expected, not very sensitive to $s$.

Changing the 'ratio of charge to neutral by 3 times its estimated error changes $\langle\epsilon>$ by $2 \%$. Another physical model studied allows for the production of $\pi, \eta, K, N$ and adjusts these, where permitted, to agree with observation; again, $\langle\epsilon\rangle$ varies by $2 \%$. In short, by constraining models to fit the extensive observations, we achieve a high degree of model independence.

3. The Total Cross Section

Before combining the data and the efficiency into a $\sigma_{m}$ we briefly discuss a possible contamination; namely, the "two-photon" 


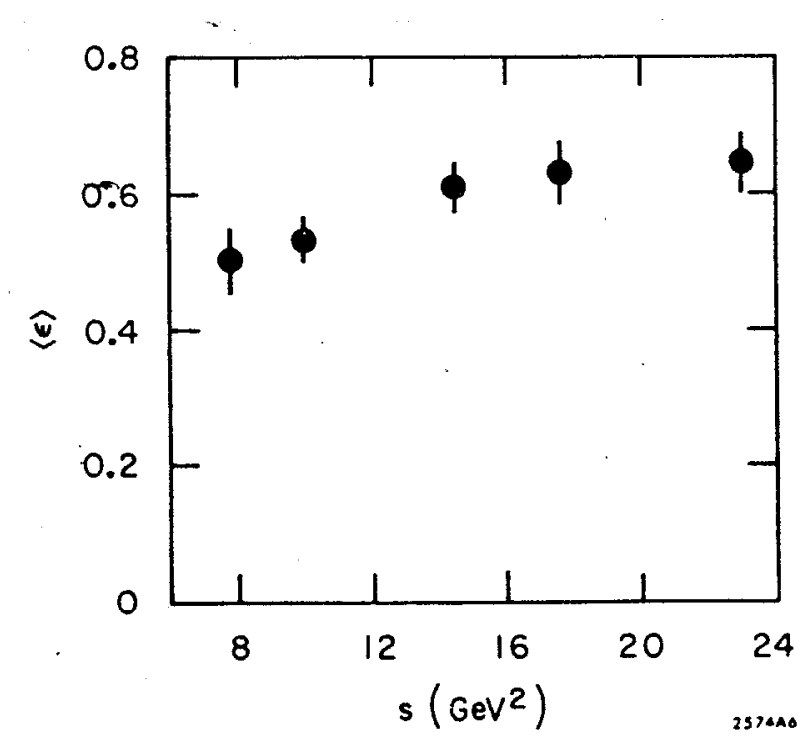

FIG. 7--Average detection efficiency from UNFOLD. (2y) cross section. (1) The process, $e^{+}+e^{-} \rightarrow e^{+}+e^{-}$ + anything, originates when two photons, emitted by the incident leptons, interact to produce the "anything." The process has an $s$ dependent cross section behaving like $\left(\log \left(\mathrm{s} / \mathrm{m}_{\mathrm{e}}\right)\right)^{2}$ (barring form factors) and overtakes single photon QED processes at 1-2 GeV energies. There are several features that mitigate against its detection in this experiment and furthermore provide a signature when it does appear: (1) the $2 \gamma$ system is dominated by small $s_{2 y}$ and therefore produces fifal state energies which are often below the detector threshold, (2) the "anything" is predominately two-body and therefore primarily coplanar with the beam line, ( 3 ) the final state $\left(\mathrm{e}^{+} \mathrm{e}^{-}\right)$have small angles relative to the beam and a fraction of them can be detected with small angle counters (the ring luminosity monitors). We have utilized (2) and (3). Two-body fincl siates in the detector are required (after analysis) to have an acoplanarity, $\Delta \phi$, greater than $20^{\circ}$. Losses of single photon annihilation to two charges with $\Delta \phi<20^{\circ}$ are restored by extrapolating the remaining distributions to $\Delta \phi=0$. The number of multihadron events that may be due to $2 \gamma$ processes is evaluated as ( $I \pm 5) \%$ by comparing the fraction of Bhabha events that are accidentally tagged by the small angle counters (0.12\%) with the fraction of hadronic events tagged (0.13\%). The fraction of coplanar "hadronic" events tagged is about xlo greater, verifying our interpretation of these events.

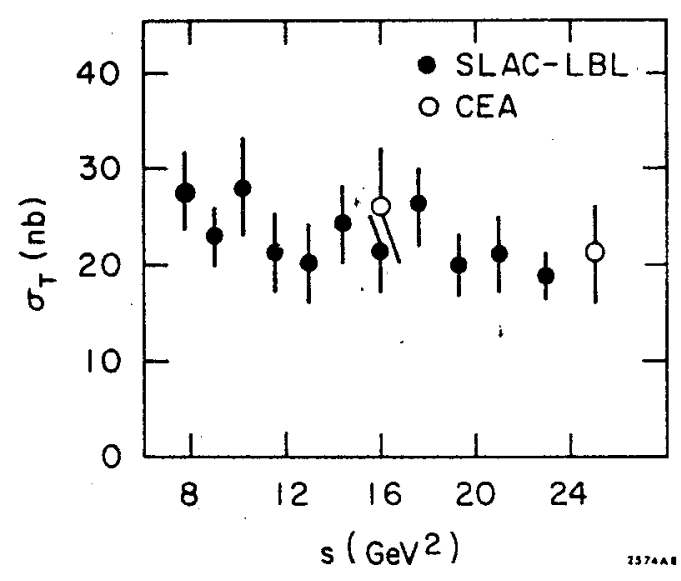

Calculable radiative effects are included in the Monte Carlo calculation of event efficiency. To account for those that are not included we have added $a \pm 5 \%$ systematic correction of $\bar{\sigma}_{\mathrm{T}}$.

$\sigma_{\mathrm{T}}$ measured in this experiment and two other measurement $s$ ) in the same $s$ range are plotted vs. $s$ in Fig. 8. The error bars include statistics and uncertainties arising from the model, $2 \gamma$ processes, radiative effects, background subtractions.

FIG. 8- $-\sigma_{\mathrm{T}}$ versus s. 
Figure 9 is an attempt to display $s \sigma_{\mathrm{T}}(\mathrm{s})$ over the energy range explored to date. The data $(2)$ is not exhaustive but is sufficient to show that when one attempts to crudely remove the photon propagator from the process the resultant "form factor" stays within the same order-of-magnitude range from $s=0.5 \mathrm{GeV}^{2}$ to $\mathrm{s}=25 \mathrm{GeV}^{2}$. The reader will note that $\mathrm{s} \sigma\left(\mathrm{e}^{+}+\mathrm{e}^{-} \rightarrow \mu^{+}+\mu^{-}\right)=87 \mathrm{nb}-\mathrm{GeV}^{2}$; the ratio $\sigma_{\mathrm{T}} / \sigma_{\text {is }}$ iscaled in Fig. 9. It is interesting that in this entire range, time-like photons couple to hadrons more strongly than to any other state.

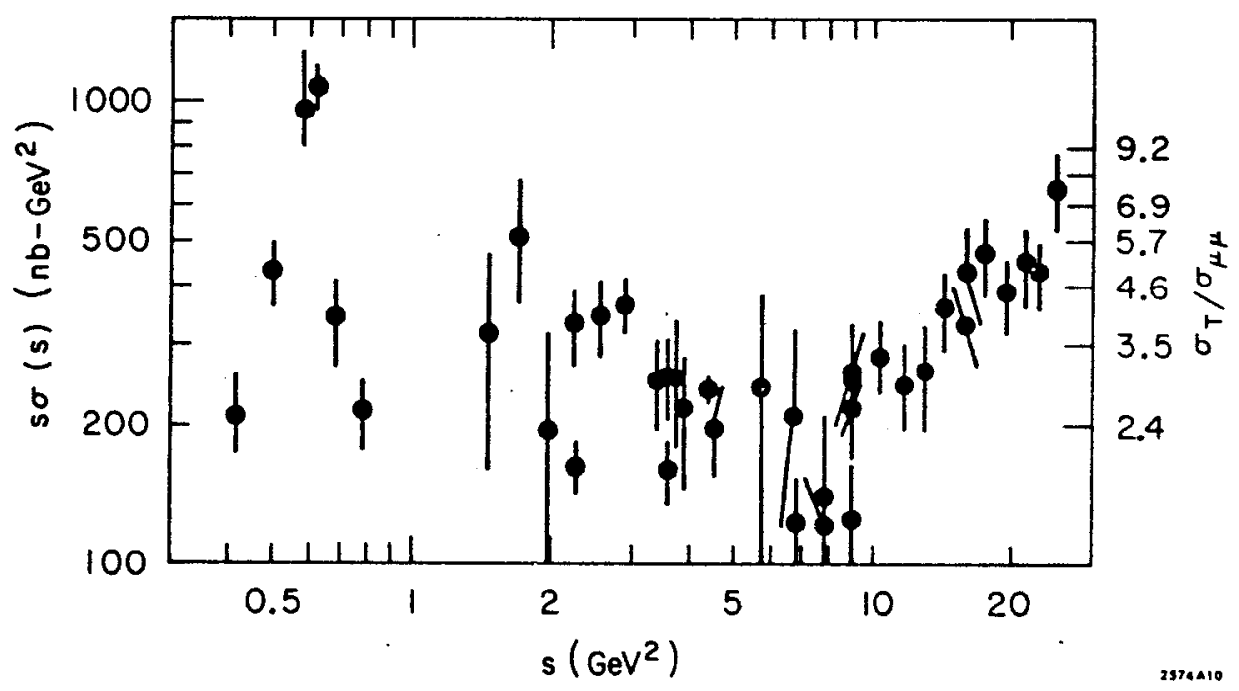

FIG. 9--s times the hadronic cross section

IV. GENERAL FEATURES OF THE FTNAL STATE

\section{Angular Distribution of Charged Particles}

All distributions observed are essentially isotropic independent of $s$ and the particle momentum, p. As an illustration, Fig. 10 is a plot of these distributions at $s=23 \mathrm{GeV}^{2}$ for two ranges of the variable $x=2 p / s^{l} / 2$. The acceptance is uniform out to $|\cos \theta|=0.6$. The data has been fit to a form $I+\alpha \cos ^{2} \theta$ and the deduced values of $\alpha$ are contained in Table II.

\begin{tabular}{|c|c|c|}
\hline $\mathbf{s}(\mathrm{GeV})^{2}$ & $\alpha(0<x<0.45)$ & $\alpha(0.45 \leqslant x<0.9)$ \\
\hline 9 & $0.0 \pm 0.17$ & $0.2 \pm 0.4$ \\
\hline 14.4 & $0.18 \pm 0.17$ & $0.0 \pm 0.4$ \\
\hline 23 & $0.25 \pm 0.1$ & $0.2 \pm 0.4$ \\
\hline
\end{tabular}




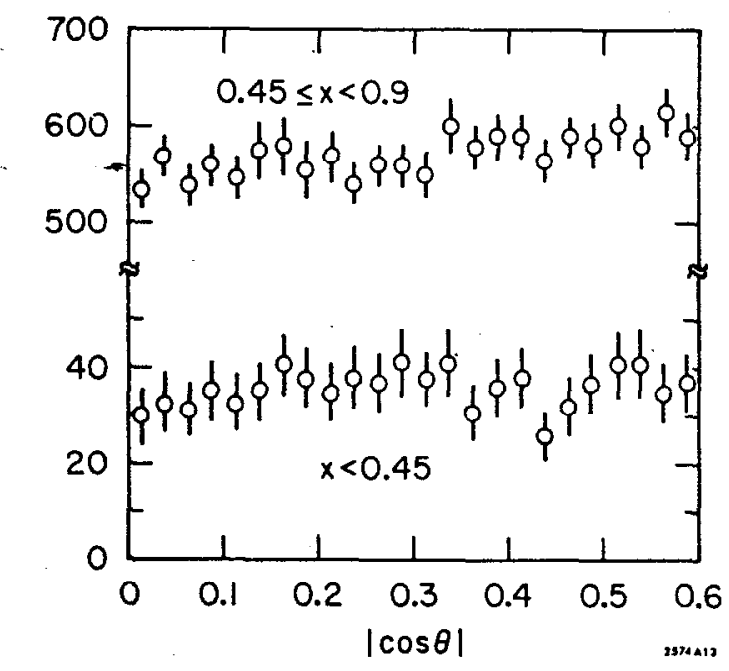

FIG. 10--Angular distribution vs. $|\cos \theta|$ at $s=23 \mathrm{GeV}^{2}$.
In a recent extension of the magnetic detector, spark chambers were added that cover an angular range of $0.87 \leqslant \cos \theta$ $\leqslant 0.94$. While the data is still under analysis the yield $d \mathrm{~N} / \mathrm{d} \cos \theta$ does not appear to be greater than that in the region $\mid \cos \theta<$ 0.6 .

\section{Averaged Parameters of the Final State}

Figure 11 displays $\left\langle\mathrm{n}_{\mathrm{ch}}\right\rangle$ $\langle p\rangle$ and $\left\langle n_{\mathrm{ch}}>p\right\rangle / \mathrm{s}^{l} / 2_{\mathrm{Vs}}^{\mathrm{ch}} \mathrm{s}_{\text {. }}$ It is noteworthy how slightly these features change as the $s$ changes by a factor of $\sim 2.5$. If one assumes pion-only production and calculates $\left\langle\mathrm{n}_{\mathrm{Ch}}>\left\langle\mathrm{E}_{\pi}>\right.\right.$

divided by the available center-of-mass energy radiatively corrected then this parameter has a slightly higher value than $\left\langle n_{c h}>p>/ s^{1} / 2\right.$ and the same $s$ dependence. The implied gradual increase (with $s$ ) in the fraction of energy going into neutrals would damage a model of exclusively pion production in the $I=0$ state.

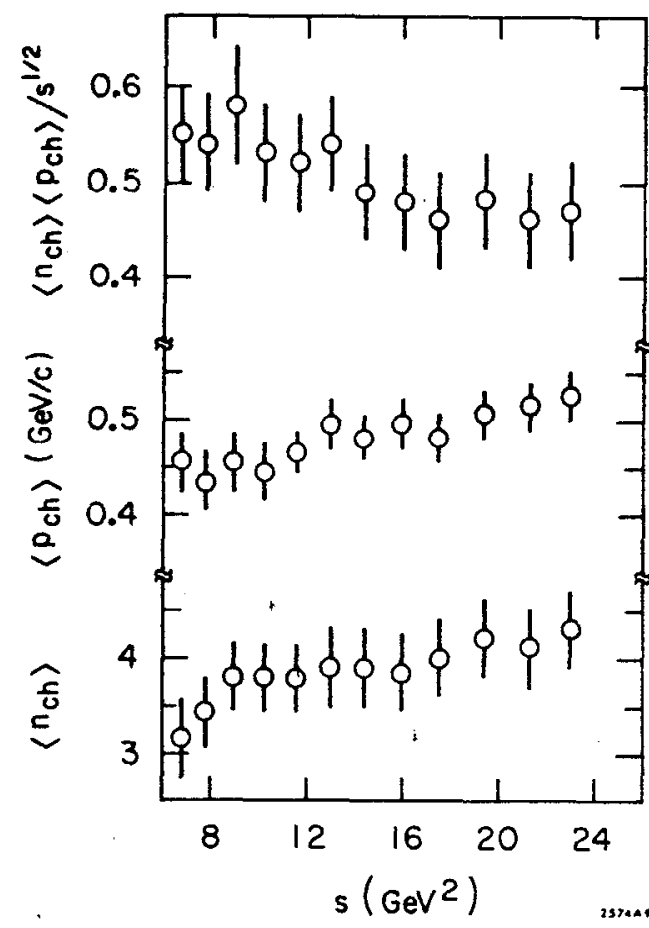




\section{Kaon and Nucleon Production}

Measuring the momentum and time of flight from the interaction region to the trigger counters provides a technique for identifying $\mathrm{K}^{\prime} \mathrm{s}$ up to about $0.6 \mathrm{GeV} / \mathrm{c}$ and nucleons up to $1.2 \mathrm{GeV} / \mathrm{c}$. Translated into total energy this corresponds to $\mathrm{M}_{\mathrm{K}} \leqslant \mathrm{E}_{\mathrm{K}} \leqslant 0.75 \mathrm{GeV}$ and $\mathrm{M}_{\mathrm{N}} \leqslant$ $\mathrm{E}_{\mathrm{N}} \leq 1.5 \mathrm{GeV}$.

The relative yield of $\pi^{-}, K^{-}, \bar{p}$ are, within statistics independent of $s\left(9 \leqslant s \leqslant 23 \mathrm{GeV}^{2}\right)$ over the momentum range of identification. Figure 12 displays the ratio of $\pi^{-}, \mathrm{K}^{-}, \overline{\mathrm{p}}$ to all negatives at $s=23 \mathrm{GeV}^{2}$. The trends of the data are apparent. The results are reminiscent of the $\pi: K: p \simeq$ 100: 10: I produced in hadron-hadron interactions. In particular, the momentum dependence of the fractions is quite consistent with those observed $(3)$ at the CERN ISR in the corresponding range of $p_{\perp}$.

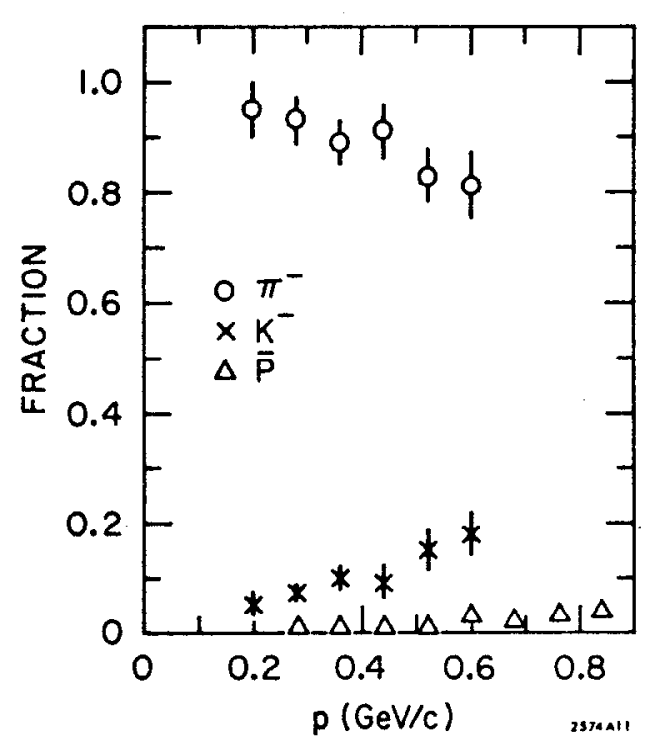

FIG. 12--Fractions of negative particles produced as $\pi^{-}, K^{-}$, $\bar{p}$ at $s=23 \mathrm{GeV}^{2}$.
Conyerting to an invariant yield $\mathrm{Ed}^{3} \mathrm{~N} / \mathrm{dp}^{3}$ and plotting vs. $E$ one obtains Fig. 13. The data is intriguing and provides great motivation to the experimenter to increase the momentum range of mass identification.

A clear $K_{0 s}$ signal can be seen in the $\pi^{+} \pi^{-}$mass distribution displayed in Fig. 14. While the final answer is not yet in on the production of $\mathrm{K}_{\mathrm{O}}$, we can state that the yield is within a factor of 2 of the charged $\mathrm{K}^{-}$yield at $s=23 \mathrm{GeV}^{2}$. This study provides an opportunity to extend the momentum range of $\mathrm{K}$ identification because it does not depend on the time-of-flight system.

\section{INCLUSIVE SPECTRA AND SCALING}

We commence this discussion with a display of the invariant cross section $\mathrm{Ed}^{3} \sigma / \mathrm{dp}^{3}$ vs. p for $s=9,14.4$ and $23 \mathrm{GeV}^{2}$ (Fig. 15). The results are essentially independent of $\mathrm{s}$ for $\mathrm{p} \lesssim I \mathrm{GeV} / \mathrm{c}$ and then appear to diverge. Before jumping to any conclusions one should recall that the kinematic limits for the 3 different values of $s$ are at $p_{\max } \simeq 1.5,1.9$ and $2.4 \mathrm{GeV} / \mathrm{c}$ and the divergence is possibly a reflection of kinematic limits on the phase space. The straight lines in Fig. 15 bound the distributions obtained in 200-300 GeV proton-nucleon collisions at $90^{\circ}$ in the center of mass $(4)$, normalized to our data. Before proceeding let's recognize a striking general feature of $\mathrm{e}^{+} \mathrm{e}^{-} \rightarrow$ hadrons in this s range: $\left\langle\sigma_{\mathrm{T}}\right\rangle,\left\langle\mathrm{n}_{\mathrm{ch}}\right\rangle,\langle\mathrm{p}\rangle, \mathrm{Ed}^{3} \sigma / \mathrm{dp}^{3}$, 
angular distributions and the observed fractions of $\pi, K, \mathbb{N}$ are remarkably insensitive to $\mathrm{s}$. These parameters are changing by about $10 \%$ while $\mathrm{s}$ varies by $\sim \mathrm{x2.5}$. Except for the magnitude of $\sigma_{\mathrm{m}} \sim 10^{-6}$ $\mathrm{x} \sigma$ (hadron + hadron) you'd swear that SPEAR was a hadron storage ring.

The most daring theoretical proposal that preceded this experiment was based on the Bjorken scaling hypothesis. (5) Introducing the dimensionless variable $x=2 P \cdot Q / Q \cdot Q][P(Q)$ is the hadron (photon) 4-vector] which is the hadron energy relative to its maximum value, $2 \mathrm{~F}_{\mathrm{f}} / \mathrm{s}^{\mathrm{I}} / 2$, it was predicted that the inclusive hadron spectra would have the form

$$
s \frac{d \sigma}{d x}=f(x),
$$

(scaling

for any species of hadrons. Integrating one has

$$
\mathrm{s}<\mathrm{n}>\sigma_{\mathrm{T}}=\int \mathrm{f}(\mathrm{x}) \mathrm{dx}=\text { constant }
$$

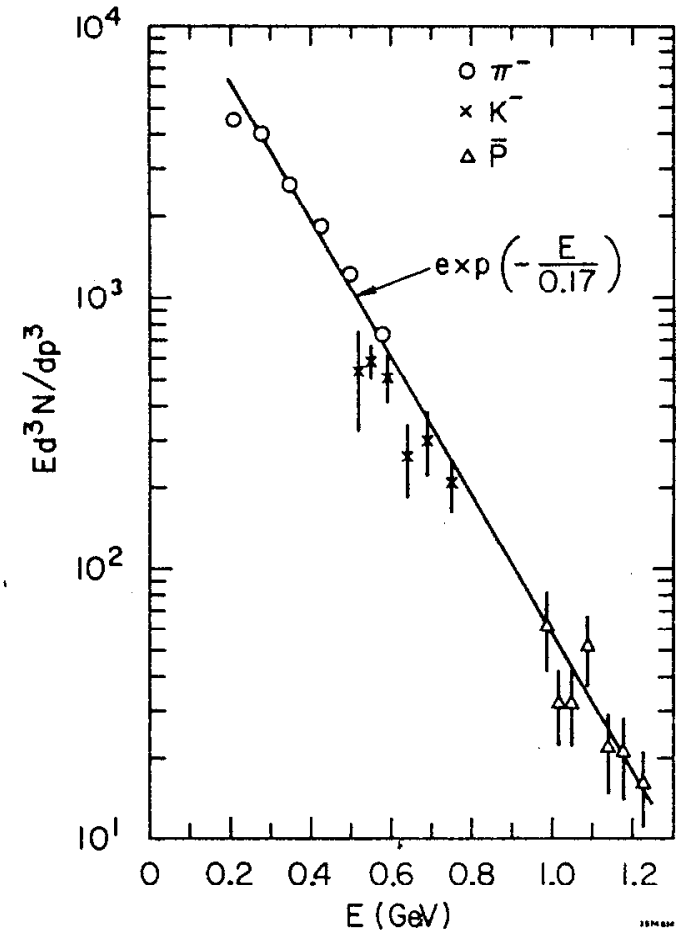

FIG. 13--Invariant yield of $\pi^{-}, K^{-}, \bar{p}$ vs. energy at $\mathrm{s}=23 \mathrm{GeV}^{2}$.

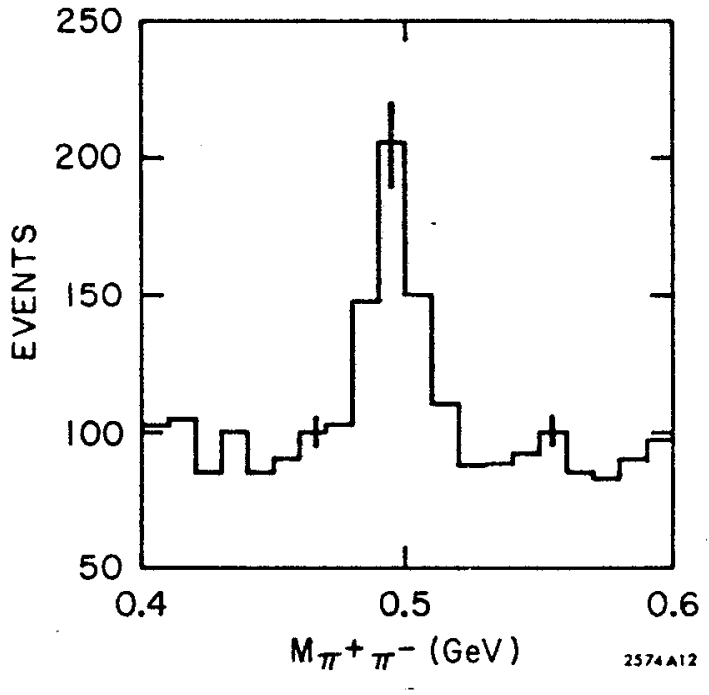

FIG. 14--Invariant mass distribution of $\pi^{+} \pi^{-}$pairs at $s=23$ $\mathrm{GeV}^{2}$. 
From the observations of the previous paragraph, it is apparent that scaling of this type is far from reality in this s range. Nevertheless, in homage to the ingenuity of our theoretical colleagues, we investigate the validity of $(1)$ by plotting $s(d \sigma / d x)$ vs. $x$ for $s=\overline{9}, 14.4$ and $23 \mathrm{GeV}^{2}$ (Figs. 16, 17). Figure 16 shows that scal. ing is grossly violated for $x \leqslant 0.5$ and Fig. 17 shows it prevails operationally to the $10 \%$ level for $x \geqslant 0.5$. However, it will be important to determine how much of this scaling is of a dynamic origin as distinguished from that which might arise from kinematic constraints and/or phase-space limitations. The author notes that this is contrary to what one might have naively expected because the deep inelastic region is at small $x$ and $x \rightarrow I$ is approaching the elastic limit. Attempts $(6)$ to salvage some form of a constituent model by the introduction of additional parameters have led to predictions that $\left(I / \sigma_{T}\right) d \sigma / d x$ should be independent of $\mathrm{s}$. Figure 8 is persuasive evidence that this notion is incorrect.

FIG. 15--Invariant differential cross section vs. $p$ for 3 values of $s$.
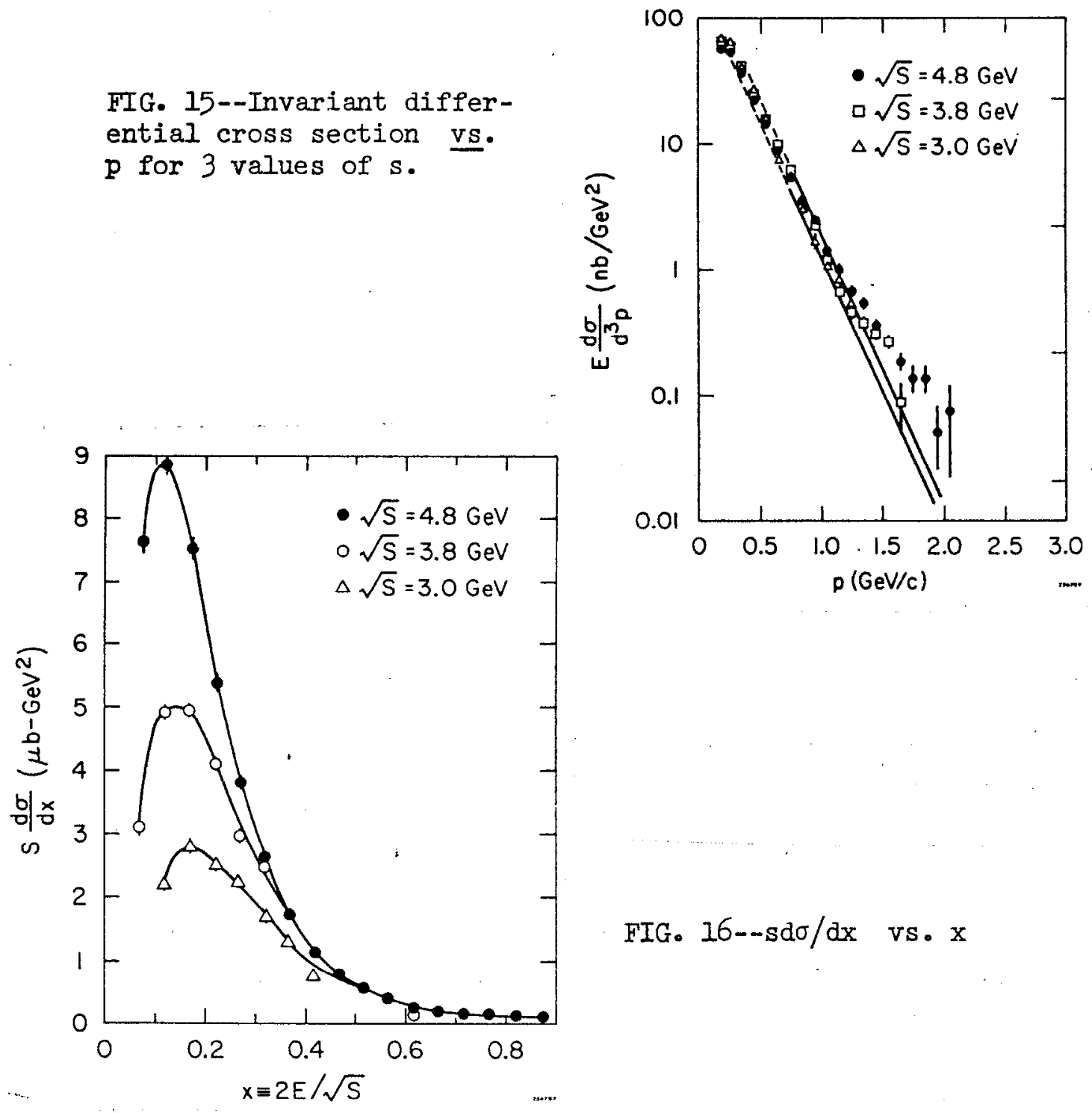

FIG. $16--s d \sigma / d x$ vs. $x$ 


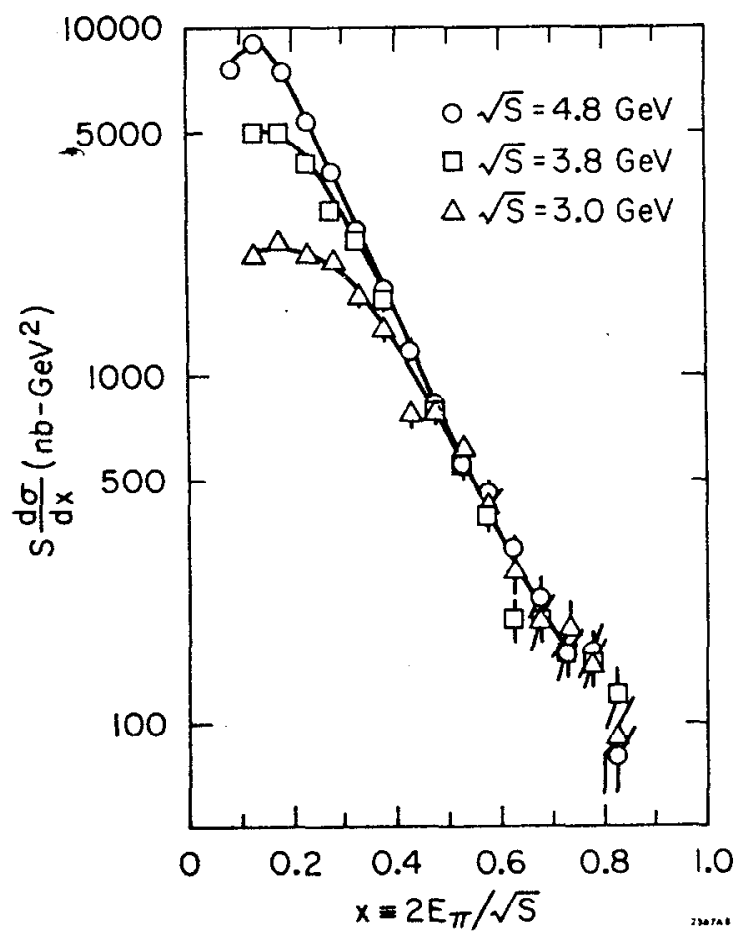

FIG. $17--\operatorname{sd} \sigma / d x$ vs. $x$

FIG. 18-- $\frac{I}{\sigma_{T}} \frac{\mathrm{d} \sigma}{\mathrm{dx}}$ for $\mathbf{s}=9,14.4,23 \mathrm{GeV}^{2}$.

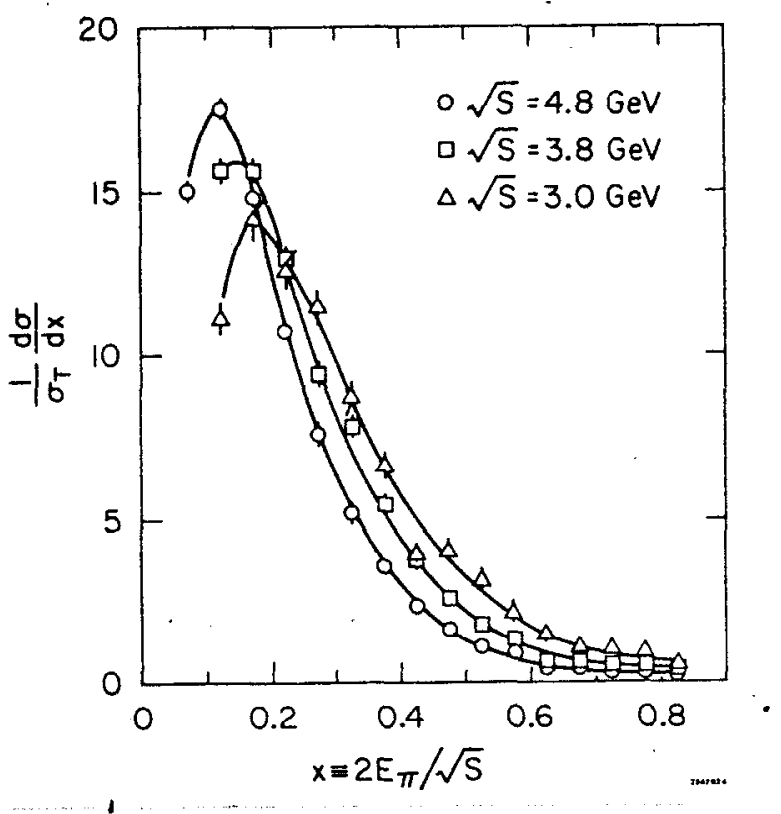


IV. CONCLUSIONS AND FUTURE DIRECTIONS

- The author's opinion is that the salient feature of $\mathrm{e}^{+} \mathrm{e}^{-}$ annihilation into hadrons is the $s$ independence of the general features of the final state. Aside from the magnitude of $\sigma_{T}$, no conspicuous aspect arises to distinguish the process from what has been observed for many years in hadron-hadron interactions with the qualification that $\mathrm{e}^{+} \mathrm{e}^{-}$annihilation apparently manifests no leading particle effects.

With the advent of DORIS (DESY) and the current energy improvement program at SPEAR, we will soon be acquiring new knowledge up to $s \simeq 70 \mathrm{GeV}^{2}$. Additionally, attempts will be made to extend the momentum range of mass identification. In view of the disarray of our understanding, any results will be of great interest.

\section{REFERENCES}

1. H. Terazawa, Rev. Mod. Phys. 45, 615 (1973).

2. K. Strauch, Proc. of the VIth Int. Sym. on Electron and Photon Interactions; M. Bernardini, et al., CERN Prepring; Submitted to Physics Letters; G. Tarnopolsky, et al., Phys. Rev. Lett. 32, 432 (1974); J. Perez-y-Jorba, IVth Int. Sym. on Electron and Photon Interactions; J.-E. Augustin, et al., Phys. Lett. 28B, 508 (1969); M. Grilli et al., Nuovo Cimento, Vol. 13A N.3 (593) 1973.

3. B. Alper et al., Phys. Lett. 47B, 275 (1973).

4. J. W. Cronin et al., Phys. Rev. Lett. 31, 1426 (1973).

5. J. D. Bjorken, Current Algebra at Smal7 Distances, Varenna School Irectures, Course XII, Varenna, Italy, 1967. S. D. Drell, D. J. Levy, T. M. Yan, Phys. Rev. 187, 2159 (1969); DI (1970) 1035.

6. G. B. West, P. Zerwas; Report Number SLAC-PUB 1420 (SLAC) 1974. 